\title{
Nahaufnahmen
}

\section{Chick Corea (1941-2021)}

\author{
Michael Rüsenberg ${ }^{*_{1}}$
}

[1] jazzcity.de, Köln, Deutschland.

\section{Zusammenfassung}

Im Gegensatz zu den meisten Jazzmusiker*innen heute, insbesondere denen in Europa, hat Chick Corea nie eine Musikhochschule besucht. Aber er wird dort studiert, denn er hat geradezu im Überfluss das, woran es vielen mangelt: eine eigene Handschrift, einen eigenen Personalstil. Dass er diesen in verschiedenen Feldern zum Ausdruck brachte, wurde oft ehrenvoll, aber fälschlich, mit dem Attribut „Chamäleon“ umschrieben. Denn niemals ist er im jeweiligen Kontext verschwunden, sondern hat ihm jeweils seinen Stempel aufgeprägt vom Klavierkonzert mit den Londoner Sinfonikern bis zu seinen „Children Songs“, solo. Wo Corea drin war, hat man es herausgehört. Das meiste davon im Jazz: sein Ton, seine rhythmisch-melodischen Signaturen auf Piano, E-Piano und monophonem Synthesizer, häufig in staccato Phrasierung, gehören zu den Ikonen der gesamten Gattung. Corea war erfolgreich, ohne Zweifel. Er hat mit seinen Improvisationen und Kompositionen Heerscharen von KollegInnen zum Imitieren und Interpretieren angeregt („Spain“, „La Fiesta“ und und und...). Ihm wurden - bei 67 Nominierungen - mehr Jazz-Grammys (25) verliehen als jedem anderen aus seiner Zunft. Jüngst erst zeichnete inn eine Auswahl internationaler Kritiker im Fachmagazin „DownBeat“ in drei Kategorien aus: als Künstler, Pianist und Keyboardspieler des Jahres (2021). Gleichwohl, unter der Handvoll Millionseller des Jazz findet sich keiner seiner rund einhundert Tonträger. Armando Anthony Corea, genannt Chick, geboren am 12. Juni 1941 in Chelsea/Massachussets, ist am 9. Februar 2021 in Tampa/Florida an einer erst kürzlich bei inm diagnostizierten Krebserkrankung gestorben. Er wurde 79 Jahre alt.

\begin{abstract}
Chick Corea, unlike most jazz musicians today, especially those in Europe, never went to a music conservatory. But his work is studied there, because he has in abundance what many lack: his own handwriting, and his own personal style. The fact that he expressed this in different fields was often honorably, but falsely, described with the attribute "chameleon". Falsely, because he never disappeared into the background of the respective context. Whether it was a piano concerto with the London Symphony Orchestra or his "Children Songs", solo, he has left his own mark. If Corea was involved, it was impossible to not hear it. Mostly, this happened in jazz: among the icons of the entire genre are his tone, his rhythmic-melodic signatures on piano, electric piano and monophonic synthesizer, often in staccato phrasing. He was successful, without a doubt. With his improvisations and compositions, he has inspired a great number of colleagues to imitate and interpret ("Spain", "La Fiesta" and the list goes on...). For his work he had been nominated for 67 Jazz Grammys and has been the recipient of 25 - more than anyone else in his trade. A selection of international critics recently honored him in three categories in the specialist magazine "DownBeat": as artist, pianist and keyboard player of the year (2021). Looking at his success, it is surprising that none of his around hundred recordings can be found among the handful of million-sellers in jazz. Armando Anthony Corea, known as Chick, born on June 12, 1941, in Chelsea/Massachussets, died on February 9, 2021 in Tampa/Florida of a cancer he was recently diagnosed with. He was 79 years old.
\end{abstract}

Jahrbuch Musikpsychologie, 2021, Vol. 30: Musikpsychologie - Empirische Forschungen - Ästhetische Experimente, Artikel e101, https://doi.org/10.5964/jbdgm.101

Eingereicht: 2021-06-09. Akzeptiert: 2021-11-04. Publiziert (VoR): 2021-11-18.

*Korrespondenzanschrift: Landgrafenstr. 103a, 50931 Köln, Deutschland. E-Mail: post@jazzcity.de

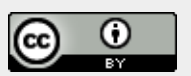

Dieser Open-Access-Artikel steht unter den Bedingungen einer Creative Commons Namensnennung 4.0 International Lizenz, CC BY 4.0 (https://creativecommons.org/licenses/by/4.0/deed.de). Diese erlaubt für beliebige Zwecke (auch kommerzielle) den Artikel zu verbreiten, in jedwedem Medium zu vervielfältigen, Abwandlungen und Bearbeitungen anzufertigen, unter der Voraussetzung, dass der Originalartikel angemessen zitiert wird.

Für das Frühjahr 2021 war eine kleine Europa-Tournee angesetzt, u.a. mit der Station Wiener Konzerthaus, Pandemie-bedingt die Wiederholung eines bereits zweimal verschobenen Konzertes von Chick Corea mit seinem neuen Trio Vigilette (Carlitos Del Puerto, b, Marcus Gilmore, dr). Ob er auch diesmal sein Publikum 
nicht nur entzückt, sondern auch aktiv (heute muss man sagen „pro-aktiv“) einbezogen hätte? Etwa durch Vorgabe des Kammertones „a“ wie auf seinem letzten, zu Lebzeiten veröffentlichten Albums „Chick Corea plays..."? Vermutlich. Wir haben es erlebt, bei der Chick Corea Akoustic Band, einem seiner bedeutenden Langzeitprojekte (mit John Patitucci, b, Dave Weckl, dr) in der Philharmonie Essen. Der Pianist wickelte, nahezu wortwörtlich, das Publikum um die Finger. Eine der größten Stimmen der Jazzgeschichte, dem das Auditorium sowieso zu Füßen liegt, griff zu Mitteln aus dem Varieté. Eben das macht die Dualität, man könnte auch sagen: die Facetten dieses Musikers aus. Sein Ton, seine rhythmisch-melodischen Signaturen auf Piano, E-Piano und monophonem Synthesizer, häufig in staccato Phrasierung, gehören zu den Ikonen der gesamten Gattung.

Ihr Einfluss auf die Formsprache des Jazz - noch dazu in verschiedenen Stilen - ist gigantisch. Wobei „einflussreich“ nicht unbedingt mit „erfolgreich“ kongruent ist (wie bei seinem Freund und Partner Herbie Hancock): der Mann hat Erfolg gehabt, ja, zuhauf. Er hat mit seinen Improvisationen und Kompositionen Heerscharen von KollegInnen zum Imitieren und Interpretieren angeregt („Spain“, „La Fiesta“ und, und, und...), ihm wurden - bei 67 Nominierungen - mehr Jazz-Grammys (25) verliehen als jedem anderen aus seiner Zunft. Aber unter der Handvoll Millionseller des Jazz findet sich keiner seiner rund einhundert Tonträger. Auch sein außer-musikalischer Einfluss wurde gelegentlich als beängstigend eingestuft; zum Beispiel in Baden-Württemberg 1993, wo die Landesregierung einem Veranstalter in Stuttgart den Subventionshahn zuzudrehen drohte und der Künstler vor dem Verwaltungsgericht Mannheim mit seinem Einspruch scheiterte. Grund war Corea's Bekenntnis zu Scientology. Seine Fans nahmen dies ebenso billigend in Kauf wie die Lobhudeleien auf und die Performances mit seiner Ehefrau Gayle Moran. Those were the days. Der Ministerpräsident damals hieß Erwin Teufel. Sein Nachnachfolger Kretschmann säße heute bei Chick Corea in Stuttgart in der ersten Reihe. Seit der Trauerfeier für Wolfgang Dauner (1935-2020) wissen wir von der Jazz-Affinität des grünen MP. Aber sollte er in seiner Chick Corea-Begeisterung womöglich auf jenen Begriff verfallen, der in den Nachrufen durch die Feuilletons fliegt (Chick Corea als „Chamäleon“), sähen wir uns zur Opposition gezwungen. Denn was auch immer Corea angefasst hat, es ist nicht im jeweiligen Kontext verschwunden, sondern er hat ihm einen, nämlich seinen Stempel aufgeprägt - vom Klavierkonzert mit den Londoner Sinfonikern bis zu seinen „Children Songs“, solo. Wo Corea drin war, hat man inn herausgehört.

Armando Anthony Corea, genannt Chick, geboren am 12. Juni 1941 in Chelsea/Massachussets, einem Vorort von Boston, hat - im Gegensatz zu heutigen Jazzmusikern - nie ein Studium absolviert. Seine einzige formale Ausbildung, sagt er in einem Interview, seien sechs Jahre Unterricht ab dem 8. Lebensjahr bei Salvatore Sullo gewesen, einem Konzertpianisten in Boston. Seine Jazz-Edukation war noch informeller, nämlich durch seinen Vater Armando, einen Amateurjazzmusiker, mit dem er früh auch aufgetreten ist. Sein erstes Jazzvorbild: Horace Silver. 1962 beginnt Coreas professionelle Karriere in New York, bei Mongo Santamaria, es folgen Jobs bei Stan Getz, Herbie Mann u.a. Bereits sein zweites eigenes Album „Now he sings - now he sobs“ setzt 1968 Standards: für seine spezifische Ausformung des Jazztrio-Formates (damals mit Miroslav Vitous, b, und Roy Haynes, dr), für einen Pianostil, dem nichts mehr von Horace Silver anhaftet. Und sein Faible für das Spanische: der opener von „Now he sings..." (Steps - what was“) enthält bereits die Blaupause für „Spain“, einen seiner großen Erfolge fünf Jahre später. Im September 1968 steigt Corea für Herbie Hancock bei Miles Davis ein („Filles des Kilimanjaro“), die wenigen FreeJazz-Momente von Miles verbinden sich wenig später insbesondere mit den Passagen, wo unter seinen schrill perlenden Läufen auf dem Fender Rhodes Electric Piano der Beat aufgerieben wird. Seine Konversion zur wirklichen Avantgarde, 1970 im Quartett Circle (u.a. mit Anthony Braxton, as), war kurz und von langfristiger Konsequenz. Im Gegensatz zu dem, was jetzt manche 
Nachrufe insinuieren, missfiel inm dieser Ansatz außerordentlich, er hielt ihn für „zu unvorhersehbar“. Es drängte in ihm nach mehr Planung, nach - Komposition. Darunter verstand er, bestimmte Errungenschaften der klassischen Tradition - etwa das Formbewußtsein ihrer Komponisten wie auch die Disziplin ihrer Interpreten, namentlich der Sinfonieorchester - mit der Tanzbarkeit, aber auch Lockerheit von Jazz und folkloristischer Musik zu verbinden. Die erste Ausformulierung dieser Prinzipien war die "halb-elektrische“ Formation von Return To Forever (u.a. mit dem Saxophonisten Joe Farrel). Man kann sie über fast alle weiteren Stationen seiner Karriere, vielfach unter Zumischung des „Spanischen“ und „Brasilianischen“, in verschiedenen Genres wiedererkennen: von seinen solistischen „Childrens Songs“ über die diverse Jazzrock-Bands bis zu seinen Orchesterwerken. Nicht alle gelungen, manche mit Pomp, manche bloße Fingerübungen, ja manches auch kitschig. Aber, viele, viele ,nuggets' (Reinhard Kopiez), great moments of jazz history. Chick Corea hatte ein Händchen für Rhythmusgruppen: angefangen mit Miroslav Vitous und Roy Haynes; vieles, aber nicht alles mit Stanley Clarke, aber fast alles und über den längsten Zeitraum mit John Patitucci und Dave Weckl, egal ob „akustisch“ oder „elektrisch“. Nicht zu vergessen in den letzten Jahren Christian McBride, b, und Brian Blade, dr, sowie die ultra-kompakten Jimmy Earl, bg, und Gary Novack, dr, Anfang der 90er Jahre. Sowie da pflichten wir der FAZ bei - der „unwiderstehliche“ Steve Gadd, dr. Unvergessen sein Duo mit Corea an den keyboards in „Spanish Fantasy, II“, 1976.

Was bleibt? Posthum ist ein neues Doppel-Album der Akoustic Band erschienen, der Live-Mitschnitt eines Konzertes vom 13. Januar 2018 in St. Petersburg/Florida, Auftakt jener Tournee, die das Trio im Juli auch nach Europa führte. Für die Ausgelassenheit dort, wie sie sich in Publikums-anbiederndem sing-along zeigte (siehe oben), war in Florida kein Platz. (Wir wären aufgesprungen für Momente von Eleganz \& Dringlichkeit wie hier in „You and the Night and the Music“). Viele Tonträger werden Maßstab bleiben, ganz zu schweigen von den Tonnen, die es im Internet zu heben gilt. Und schon geht es los: Freunde schicken Youtube-links!

Armando Anthony "Chick“ Corea ist am 9. Februar 2021 in Tampa/Florida an einer erst kürzlich bei ihm diagnostizierten Krebserkrankung gestorben. Er wurde 79 Jahre alt.

\section{Finanzierung}

Der Autor hat keine Finanzierung zu berichten.

\section{Interessenkonflikte}

Der Autor erklärt, dass keine konkurrierenden Interessen bestehen.

\section{Danksagung}

Der Autor hat keine Unterstützung zu berichten.

\section{Originalitätserklärung}

Der vorliegende Text ist eine modifizierte Übernahme eines Beitrages von jazzcity.de (https://www.jazzcity.de/index.php/news/2534-chick-corea-1941-2021). Die Rechte an dem Text liegen beim Autor, eine Abdruckgenehmigung für das Jahrbuch Musikpsychologie wurde erteilt. 